\title{
Un Sistema de Enseñanza Adaptativa a Distancia Basado en Tareas y Reglas Docentes
}

\author{
(An Adaptive Distance Leaming System Based Qn Teaching Tasks and Rules) \\ ROSA MARÍA CARRO \\ ESTRELLA PULIDo \\ PILAR RODRÍGUEZ \\ E.T.S. Informática, Universidad Autónoma de Madrid \\ (España)
}

\begin{abstract}
RESUMEN: Este artículo describe cómo construir y ejecutar cursos adaptativos accesibles a través de Internet. Para ambas tareas se utiliza el sistema TA.NGOW (Task-based Adaptive learNer Guidance Qn the Web), un sistema de enseñanza adaptativa basado en la Web que guía a los estudiantes durante la realización de esos cursos. El procedimiento de guía permite que tanto los itinerarios propuestos a los estudiantes como los contenidos mostrados durante la realización de un curso se adapten al perfil del estudiante, a sus acciones durante la interacción con el curso y a la estrategia de aprendizaje activa. Este objetivo se consigue mediante la separación entre el contenido de los cursos y la estructura de los mismos, articulándose ésta última como un con junto de tareas y reglas docentes que reflejan los objetivos propuestos por el profesor.
\end{abstract}

\section{Educación a Distancia - Hipermedia adezptativa -. Generación dinámica de páginas HTML}

ABSTRACT: This paper describes how hypernedia-based adaptive courses accessible through Internet can be designed and executed. Ilie TANGOW (Task-based Adaptive learNer Guidance Qn the Web) system can be used for both pu?poses. TANGOW is an adaptive learning environment based on the Web that guides students during their learning process when following such courses. The guiding procedure allows that both the proposed learning itineraries and the contents presented to the students while interacting with the system are adaptad to the student pro file, the actions performed the student during the learning process and the selected teaching strategy. This goal is achieved by establishing a clear separation between the course contents and its structure. The course structure is defined l means of a set of teaching tasks and rules that reflect the goals proposed by the teacher.

\section{Distance Education -Adaptive Hypermedia - Dynamic HTML page generation}

\section{INTRODUCCIÓN}

El gran auge de Internet en los últimos años ha hecho posible abordar proyectos que planteen la realización de aplicaciones concretas que sean accesibles a un gran número de usuarios. En este entorno, una de las principales áreas de aplicación es la enseñanza, ya que la red permite poner a disposición de los estudiantes una gran cantidad de información a distancia.

Sin embargo, el conocimiento previo que los distintos estudiantes tienen sobre una materia puede ser muy variado, de forma que la misma página puede resultar complicada para un principiante $\mathrm{y}$, al mismo tiempo, parecer trivial y aburrida a un alumno avanzado. Por otra parte, los estudiantes principiantes entran en el hiperespacio de material educativo sin ningún conocimiento sobre el tema y la mayoría de los hiperenlaces que se les ofrecen se refieren a material completamente nuevo para ellos.

En resumen, la gran cantidad de información disponible, así como su complejidad y heterogeneidad, hacen que las herramientas de navegación existentes no sean lo suficientemente potentes como para orientar al estudiante en su búsqueda de información. Estas herramientas estándar tampoco son capaces de identificar los caminos más apropiados para cada estudiante de acuerdo con sus objetivos, sus conocimientos previos y sus necesidades. 
Una solución a este problema es dotar a los sistemas hipermedia tradicionales de la capacidad de adaptarse a las necesidades particulares de cada estudiante, adecuando las interacciones del sistema a sus características, objetivos e intereses. Un sistema de hipermedia adaptativo podría definirse entonces como un sistema de hipermedia que tiene en cuenta las características del usuario y las utiliza para personalizar la información hipermedia a la que éste accede. Desde este punto de vista, las técnicas de hipermedia adaptativo pueden ser útiles para resolver muchos de los problemas asociados al uso de hipermedia educativo.

Para completar la definición de un sistema hipermedia adaptativo es necesario especificar (1) qué características del sistema son potencialmente adaptables y (2) qué características y/o acciones del estudiante pueden utilizarse para adecuar la adaptación.

Las posibilidades de adaptación del sistema se centran en dos aspectos complementarios entre sí: la adaptación de los contenidos y la adaptación de itinerarios. La adaptación de los contenidos permitiría que la misma información se pudiera presentar con distinto grado de detalle, para estudiantes con conocimientos previos diferentes, acompañada de ejercicios de dificultad variable, etc. La adaptación de los itinerarios de aprendizaje permitiría, entre otras posibilidades, ayudar a los estudiantes modificando el orden en el que se presentan los conceptos de acuerdo con sus necesidades o preferencias.

Respecto a las características del estudiante que pueden utilizarse para dirigir el proceso de adaptación, éstas se deberían poder definir tanto asociadas a rasgos propios del estudiante como a la interacción que éste realiza con el sistema a lo largo del proceso de aprendizaje.

Entre los aspectos suceptibles de modelizar cabe citar los objetivos del estudiante, sus conocimientos previos, sus preferencias, sus necesidades, su grado de éxito en lo ya estudiado con el sistema y quizá su experiencia previa en el manejo de sistemas hipermedia.

Con estos objetivos, se ha desarrollado un sistema para la creación y seguimiento de cursos hipermedia adaptativos a través de Internet, TANGOW. En la sección 2 se detallan algunas de las principales características del sistema. En las secciones 3 y 4 se especifica cómo puede hacer uso de TANGOW un profesor y un estudiante respectivamente. La sección 5 presenta un análisis de otros sistemas existentes, y en la sección 6 se exponen las conclusiones y el trabajo futuro.

\section{2. ¿QUÉ ES TANGOW?}

TANGOW, Task-based Adaptive learNer Guidance On the Web, es un sistema que permite la creación de cursos adaptativos accesibles a través de Internet (Carro, 1999a, 2000; Suárez, 2000). Los itinerarios propuestos para el seguimiento del curso son específicos para cada estudiante, y constituyen un procedimiento de guía para la realización del curso.

Para dotar a los cursos de capacidades adaptativas, TANGOW gestiona de forma independiente la estructura de los cursos y sus contenidos. Por ello, para crear un curso es necesario definir primero su estructura conceptual y asociar después a esa estructura los contenidos que aparecerán en las páginas HTML que se presentarán a los estudiantes. La estructura se describe mediante un conjunto de tareas y reglas docentes que reflejan los objetivos propuestos por el diseñador, mientras que las páginas HTML se generan dinámicamente en el momento previo a ser presentadas a partir de la información sobre los elementos multimedia asociados a cada tarea. Por tanto, el diseñador sólo debe proporcionar dichos elementos multimedia, clasificándolos según algunas de sus características, como el idioma en que están escritos o la dificultad que supone su comprensión.

El proceso de guía llevado a cabo por TANGOW consiste en adaptar los contenidos de un curso a cada estudiante concreto, teniendo en cuenta ciertos rasgos de su perfil, las acciones que ha realizado durante su interacción con el curso y la estrategia de aprendizaje activa. TANGOW indica en cada momento el conjunto de conceptos accesibles, ofrece ejercicios para comprobar el grado de conocimiento adquirido y analiza los resultados de esos ejercicios para decidir cuáles son los temas más apropiados para estudiar a continuación.

Estas capacidades adaptativas permiten la consideración de factores tales como la capacidad de un alumno para estudiar un determinado tema, o las distintas formas posibles de aproximarse a un mismo 
concepto. La consideración de estos factores permite que el sistema pueda enseñar un mismo tema de diferentes formas, bien adecuando la presentación del tema a cada estudiante concreto, bien ofreciendo distintas alternativas a un mismo estudiante que no esté obteniendo buenos resultados durante la realización del curso.

TANGOW almacena el itinerario seguido por cada estudiante durante el proceso de aprendizaje, así como los resultados obtenidos por el mismo durante la ejecución del curso. Esta información se utiliza, entre otras cosas, para establecer los mecanismos de continuación entre sesiones consecutivas.

Estas y otras características hacen de TANGOW un sistema de enseñanza adaptativa con muchas posibilidades para la educación a distancia. A continuación, vamos a exponer con más detalle cómo un profesor puede utilizar la herramienta de diseño para crear cursos adaptativos a través de Internet y cómo TANGOW guía a los estudiantes mientras están realizando uno de estos cursos. Para ello se utilizaremos el ejemplo que se describe a continuación.

\section{UN EJEMPLO: CONVERSIÓN DE UN NÚMERO DECIMAL A BINARIO}

Para ilustrar el proceso de generación de un curso, vamos a utilizar un ejemplo sencillo. Se trata de diseñar una unidad docente, que podría ser parte de un curso más amplio, cuyo objetivo es que los alumnos aprendan a convertir números decimales en números binarios. Esta unidad debería reunir las siguientes características:

- Incluirá explicaciones teóricas, junto con ejemplos y ejercicios sobre el tema en cuestión.

- El acceso a esta unidad se permitirá sólo a aquellos alumnos que tengan conocimientos previos sobre los números binarios en general.

- Los materiales educativos disponibles estarán escritos en los idiomas español e inglés.

El procedimiento utilizado para convertir números decimales a binarios consta de dos etapas: (a) un procedimiento iterativo en el que se realizan divisiones sucesivas por 2 del número a convertir y de los sucesivos cocientes hasta llegar a un cociente igual a 1 y (b) la construcción del resultado en biliario tomando el cociente final y los restos en orden inverso al que fueron obtenidos.

A lo largo de este artículo veremos cómo puede implementarse este ejemplo mediante el sistema TANGOW y lo utilizaremos para ilustrar los aspectos más relevantes del sistema.

\section{TANGOW Y EL TUTOR}

En esta sección se describe cómo se puede utilizar el sistema TANGOW para construir cursos adaptativos accesibles a través de Internet. En lo sucesivo se asume que es el tutor el que construye los cursos, aunque éste no tiene por qué ser el caso. El proceso de construcción de un curso puede estructurarse en dos partes: (1) describir la estructura completa del curso y (2) decidir y clasificar los elementos multimedia que aparecerán como contenidos del curso.

\subsection{LA ESTRUCTURA DEL CURSO}

Para que los cursos generados puedan ser adaptativos y el sistema TANGOW pueda guiar a los estudiantes mientras éstos aprenden, la estructura de los cursos se especifica mediante un conjunto de tareas y reglas docentes. Así pues, la primera tarea del profesor es describir este conjunto de tareas y reglas para cada curso que quiera implementar.

Una tarea docente es la unidad o componente básico de un curso. Los cursos están formados por una tarea principal que normalmente está dividida en subtareas, las cuales, a su vez, pueden estar compuestas por otras subtareas, y así sucesivamente. De esta manera, un curso puede ser considerado como una tarea principal que tiene que ser llevada a cabo por los estudiantes, los cuales deberán realizar algunas o todas las subtareas que componen dicho curso. 
La descripción de una tarea incluye información sobre el nombre de la tarea, la descripción de la misma, su atomicidad (atómica o compuesta), el tipo de tarea (teoría, practica o ejemplos), y el tipo de contenidos multimedia asociados que aparecerán en las páginas HTML que se presentarán a los estudiantes. Además, puede tener definidos unos requisitos de finalización, que indican cuándo la tarea se puede considerar realizada por el estudiante. Estos requisitos de finalización dependen de las páginas que hayan sido ya visitadas por el estudiante, en el caso de tareas teóricas o de ejemplos, y de los ejercicios resueltos yio resultados obtenidos, en el caso de tareas de tipo práctico.

A su vez, una regla docente indica cómo se descompone una tarea en subtareas. Pueden definirse distintas reglas para una misma tarea compuesta, cada una de las cuales describe una forma diferente de descomponer la misma tarea. En este caso, cada regla ha de tener asociada una condición de activación distinta, que indica en qué casos se va a aplicar la regla concreta. Estas condiciones de activación pueden estar relacionadas con el perfil del estudiante o con las acciones llevadas a cabo durante el proceso de aprendizaje. Esto hace posible la coexistencia en un mismo curso de distintas formas de estudiar los mismos conceptos, disponibles para los distintos tipos de estudiantes.

Las reglas tienen otra función adicional, ya que permiten al tutor establecer dependencias entre tareas, de forma que para poder acceder a una tarea sea necesario que el estudiante haya realizado otra previamente o que haya obtenido unos resultados determinados durante la realización de la misma. De esta manera puede impedirse el acceso de un estudiante a un tema que requiera unos conocimientos previos expuestos en otra parte del curso no visitada aún por el mismo, e incluye la posibilidad de introducir repasos o más detalles de un tema concreto si el estudiante no obtuvo buenos resultados en otro tema relacionado.

Para definir una regla es necesario indicar cuál es la tarea compuesta, cuáles son las subtareas en que se descompone, el orden o secuenciación en el que éstas deben realizarse y las condiciones de activación de la regla, en caso de que las haya. Existen distintos modos de secuenciación: AND indica que deben realizarse todas las subtareas en el orden en que aparecen en la regla, ANY también indica que deben realizarse todas, pero en cualquier orden, OR permite realizar una o varias, y XOR sólo permite realizar una de ellas. Además debe describirse cómo se calculan determinados valores relativos a la tarea compuesta, como por ejemplo el número de páginas HTML correspondientes a esa tarea que el estudiante ha visitado, que suele calcularse en función de los valores correspondientes relativos a las subtareas.

Así pues, para diseñar un nuevo curso, el profesor debe identificar las unidades conceptuales que constituirán las tareas docentes, y definir mediante reglas cómo esas tareas se descomponen en subtareas. Existe una herramienta basada en páginas HTML que facilita el diseño de los cursos mediante el uso de formularios que el diseñador debe completar con las descripciones deseadas. Estas descripciones se guardan en una base de datos para ser utilizados posteriormente por el sistema.

El hecho de que las tareas, las reglas y el material multimedia se almacenen en bases de datos permite que los diseñadores puedan variar, añadir o eliminar componentes del curso fácilmente y que éstos puedan ser reutilizados en diferentes cursos.

\subsection{LOS ELEMENTOS MULTIMEDIA}

Además de especificar la estructura del curso, el tutor debe decidir el conjunto de elementos multimedia (textos, imágenes, vídeos, sonidos, animaciones, applets, ...) que utilizará el sistema TANGOW para generar dinámicamente las páginas HTML que se presentarán al estudiante. Pueden asociarse elementos de distintas características a un mismo concepto, de forma que se seleccione el más apropiado para cada estudiante dependiendo de sus características particulares. Por ello, es necesario clasificar los elementos multimedia en función de distintos criterios como el idioma en que están escritos o la dificultad de los contenidos.

Si en algún momento posterior a la creación de un curso un profesor deseara modificar alguna parte del curso, podría hacerlo de manera sencilla. El hecho de que las páginas HTML se construyan dinámicamente a partir de elementos multimedia clasificados según sus características propias, hace posible un fácil mantenimiento de la información. El profesor únicamente tendrá que acceder a aquellos elementos multimedia relacionados con el tema en cuestión y modificarlos. Pueden encontrarse más detalles sobre el proceso de diseño de cursos en Carro (1999c). 


\section{LAS TAREAS Y REGLAS DEL EJEMPLO}

En nuestro ejemplo sobre conversión de números decimales a binarios, podrían identificarse las siguientes unidades básicas o tareas atómicas:

- Explicación del procedimiento de división

- Explicación del procedimiento de obtención de los bits que formarán el número binario

- Ejemplos ilustrativos

- Ejercicios relacionados

Utilizando la herramienta de diseño disponible, el profesor deberá especificar las características de cada una de estas tareas. Esta descripción será almacenada en la base de datos y utilizada por el sistema para guiar al estudiante en su proceso de aprendizaje. En la figura 1 se muestran las definiciones de algunas de las tareas identificadas.

\begin{tabular}{|c|c|c|c|}
\hline NAME & D2B_thl & NAME & DZB_ener \\
\hline TYPE & Teoria & TrPE & Practaca \\
\hline ATOMAC & Si & ATOMC & Si \\
\hline E.DBSCRUPHON & 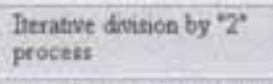 & E_DESCRIPIION & $\begin{array}{l}\text { Exerciutes about decimal } \\
\text { to binary conversion }\end{array}$ \\
\hline S_DESCRUTHON & $\begin{array}{l}\text { Divisiones sucesivas por } \\
z^{2}\end{array}$ & S_DESCRIPTION & $\begin{array}{l}\text { Ejercicios de conversion } \\
\text { de decrnal a binario }\end{array}$ \\
\hline END_METHOD & F_TEO & END_MCIHOD & F_PRAC \\
\hline PARAMS & $\begin{array}{l}\text { pags_vinited } \\
\text { tot_pags }\end{array}$ & PARAMS & $\begin{array}{l}\text { exer_ok } \\
\text { exer_done }\end{array}$ \\
\hline \multirow[t]{2}{*}{ Hmat. } & Div2 & & tot_poer \\
\hline & & HTha. & $\begin{array}{l}e 1 \mathrm{a} \\
\mathrm{e} 2 \mathrm{a} \\
\mathrm{e} 3 \mathrm{f} \\
\mathrm{ed} \mathrm{d} \\
\mathrm{e} \text { e }\end{array}$ \\
\hline
\end{tabular}

Figura 1: Ejemplos de tareas atómicas

Como muestra la figura 1, la tarea "D2B_thl” corresponde a la explicación teórica del procedimiento de división. Esta es una tarea de teoría y atómica. El método a ejecutar para comprobar si la tarea ha finalizado es "F_TEO" que recibe como parámetros el número de páginas asociadas con esa tarea (“tot_pag”) y el número de páginas visitadas por el estudiante (“pags_visited”) y comprueba que ambos valores coinciden, es decir, que el estudiante ha visitado todas las páginas relacionadas con la tarea. Finalmente, el campo HTML contiene una lista de identificadores correspondientes a los elementos multimedia necesarios para construir la página HTML. En nuestro caso esta lista tiene un único elemento: "Div2". Durante la ejecución del curso, el sistema compone la página HTML que se presentará al estudiante seleccionando del conjunto de elementos con ese nombre los que correspondan a su perfil. De forma similar se describiría la tarea "D2B_th2", correspondiente a la explicación teórica del procedimiento de obtención del número binario.

Por su parte, la tarea "D2B_exer" es una tarea práctica y atómica. En este caso, el requisito de finalización viene dado por el método "F_PRAC", que calcula el porcentaje del número de ejercicios resueltos con respecto al total y el del número de ejercicios correctamente resueltos sobre el número de ejercicios realizados, para decidir si la tarea se puede considerar como finalizada. En este caso, en el campo HTML, además de incluir información sobre los enunciados de los ejercicios, es necesario añadir la respuesta correcta a cada ejercicio.

Una posible estrategia de diseño es partir de las tareas atómicas anteriormente identificadas y estructurarlas de forma que se definan los distintos itinerarios de aprendizaje. Se puede observar que, para comenzar, tiene sentido agrupar las dos tareas relacionadas con explicaciones teóricas sobre el 
procedimiento de conversión, obteniéndose así la tarea compuesta "D2B_theory”. La definición completa de esta tarea aparece en la figura 2.

Desde el punto de vista de la enseñanza, parece lógico considerar que la tarea de teoría esté al mismo nivel que las tareas de ejemplos y ejercicios, con lo cual pueden agruparse estas tres tareas en una de más alto nivel, asociada al tema que se desea estudiar. Esta tarea ("D2B") y su descripción aparece también en la figura 2. En este caso, se incluye una pequeña introducción que será mostrada al estudiante antes de abordar las subtareas.

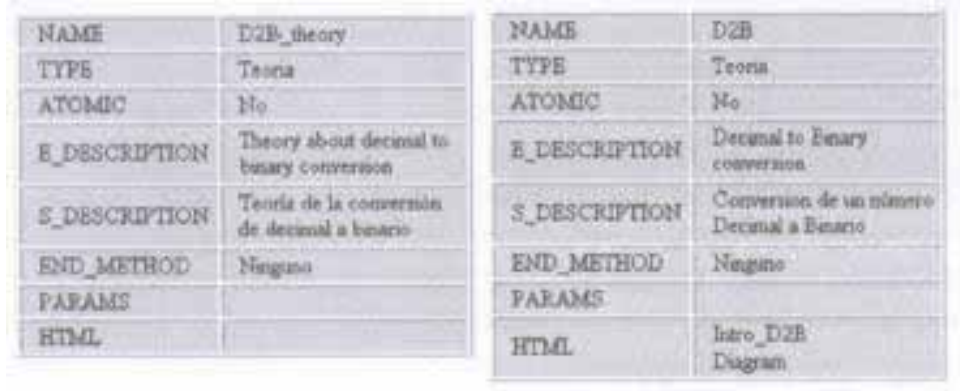

Figura 2: Ejemplos de tareas compuestas

En lo que respecta a la estructura del curso, sólo falta definir las reglas que describen la descomposición de tareas en subtareas, y que incluyen información sobre las condiciones necesarias para que el estudiante pueda realizar la tarea asociada. En nuestro ejemplo se han identificado dos tareas compuestas, con lo cual serán necesarias al menos dos reglas que las definan. La regla "Rl" describe cómo se descompone la tarea de teoría sobre conversión de números decimales a binarios, "D2B_theory", en las dos subtareas correspondientes, "D2B_thl” y "D2B_th2". El modo de secuenciación utilizado es AND, indicando que las subtareas deben realizarse en el orden especificado.

La regla "R2" (ver figura 3) describe la descomposición de la tarea principal en las subtareas de teoría, ejemplos y ejercicios. El modo de secuenciación es también AND. La regla tiene una condición de activación (método "ci”) que indica que la regla puede activarse sólo si se ha realizado previamente la tarea correspondiente a conceptos generales sobre números binarios. El método ci recibe como parámetro el valor del atributo "done" de la tarea "BINARY". Como se indicó en la sección 3.1, el campo CALC_PARAMS describe cómo se calculan determinados valores relativos a la tarea compuesta (“time_in”, “tot_pag”, “exer_ok”, ...) en función de los valores homónimos en las subtareas.

En cuanto a los elementos multimedia, el profesor debe incluir los asociados a cada una de las tareas definidas (aparecen en el campo HTML de las tareas de la figura 1). Como se mencionó previamente, los elementos deben estar disponibles en dos versiones: en español y en inglés, por lo que será necesario clasificar los elementos de acuerdo con este criterio.

\section{TANGOW Y EL ESTUDIANTE}

Para acceder al servidor de cursos TANGOW los estudiantes únicamente necesitan disponer de un navegador Web estándar. Al acceder al sistema, el estudiante debe rellenar una página inicial de identificación escribiendo un nombre de usuario, una clave, y seleccionando el curso que desea realizar. En el caso de que sea ésta la primera vez que accede a un curso, también deberá rellenar un test dónde indicará aquellas características personales que se han utilizado para determinar los perfiles de usuario, como su edad, idioma y la estrategia de aprendizaje que prefiere (teoría-antes-de-práctica o prácticaantes-de-teoría). A continuación, el sistema genera la primera página correspondiente al curso seleccionado de acuerdo con las características del estudiante. Si no es la primera vez que el estudiante accede al curso, el sistema consulta su perfil (que fue almacenado en el primer acceso) y la historia de las acciones que realizó en anteriores sesiones, reconstruyendo la situación en que se encontraba justo antes de abandonar el curso la última vez, y generando de nuevo la página que se encontraba visible en aquella ocasión. 


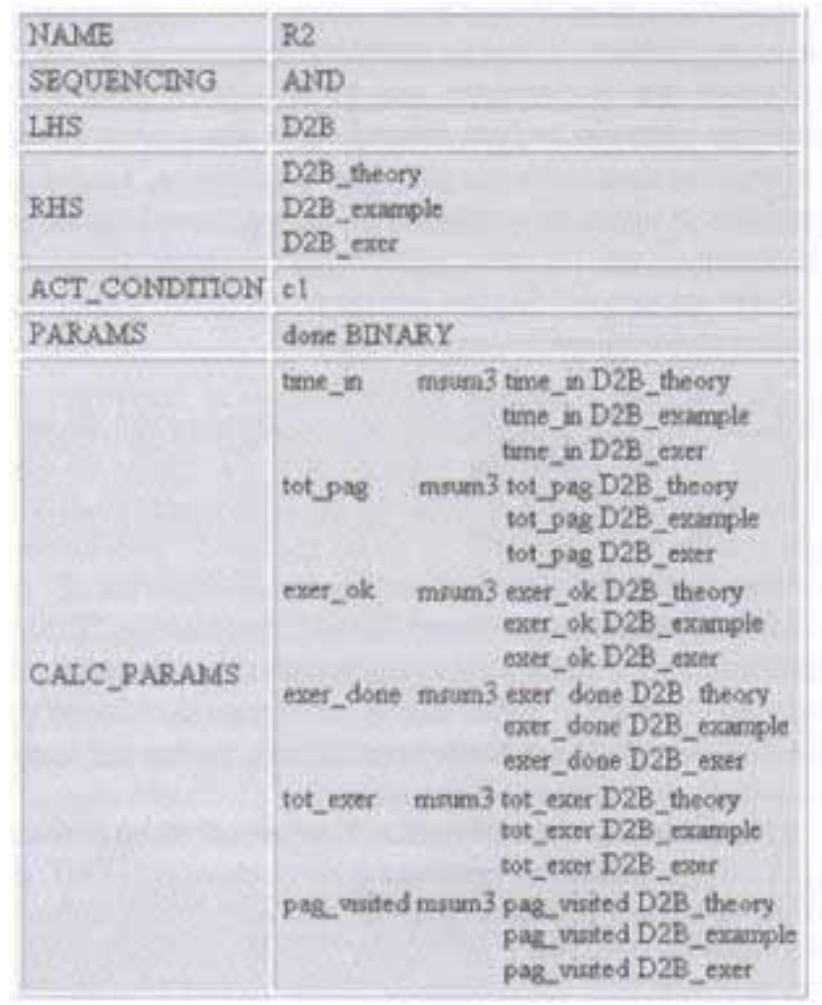

Figura 3: Ejemplos de reglas docentes

En el transcurso de cada sesión, el estudiante irá recibiendo páginas HTML con explicaciones, ejemplos o ejercicios sobre el tema objeto de estudio. La tarea del estudiante, desde el punto de vista de interacción con el sistema, es ir avanzando por el curso siguiendo las orientaciones ofrecidas, eligiendo posibles caminos en los puntos en que esto sea posible y resolviendo los ejercicios propuestos. La principal función del sistema TANGOW es guiar al estudiante durante este proceso de aprendizaje.

El proceso de guía llevado a cabo por el sistema TANGOW consiste en decidir, en cada momento, qué tareas puede o debe realizar ese estudiante concreto y cuáles no son necesarias o no pueden ser realizadas todavía por el mismo. Esta decisión está basada en: (1) la estrategia de aprendizaje que esté utilizando el estudiante, (2) las acciones previamente realizadas por el estudiante y (3) los rasgos que se han considerado significativos del perfil del estudiante. A continuación se explican cada uno de estos aspectos relacionados con la adaptatividad.

\subsection{LA ESTRATEGIA DE APRENDIZAJE}

El estudiante puede seleccionar, al comienzo de un curso, entre las dos estrategias disponibles actualmente: teoría-antes-de-práctica o práctica-antes-de- teoría. Si elige la primera, el estudiante deberá realizar primero las tareas de tipo teoría, por lo que se presentarán en primer lugar páginas HTML con contenidos teóricos. Después de estudiar la teoría, el estudiante ya tendrá acceso a las tareas de tipo práctico, pasando entonces a la realización de los ejercicios. En caso de elegir la estrategia práctica-antesde-teoría, el estudiante realizará primero las tareas de tipo práctico, intentando resolver los ejercicios en primer lugar, para pasar después a las tareas de tipo teórico, recibiendo las explicaciones teóricas relacionadas con los ejercicios resueltos.

La estrategia de aprendizaje seleccionada influye en el orden en que se presentarán al estudiante las tareas realizables cuando la secuenciación de la última regla activada es de tipo AND. Si el estudiante ha seleccionado la estrategia de aprendizaje teoría-antes-que-práctica, el orden de ejecución de las subtareas es el mismo en que aparecen en la regla; en caso de haber seleccionado práctica-antes-que-teoría, las tareas de ejercicios se propondrán antes que las explicativas.

\subsection{LAS ACCIONES DEL ESTUDIANTE}


Según las indicaciones del diseño, la activación de una regla, que da lugar a la descomposición de una tarea en subtareas, puede depender de las acciones previamente realizadas por el estudiante. El sistema tiene en cuenta estas acciones en el momento de evaluar qué reglas están activas en un momento concreto. Cuando un estudiante aborda una tarea que está definida como tarea compuesta, el sistema ha de buscar qué reglas indican posibles descomposiciones de esa tarea y evaluar sus condiciones de activación. Las reglas cuya condición de activación se satisfaga determinan el conjunto de subtareas que deben ser realizadas por el estudiante para considerar la tarea compuesta como realizada.

Durante la interacción del estudiante con el curso, TANGOW va almacenando los resultados de sus acciones, es decir: el número de páginas visitadas, el número de ejercicios resueltos, el número de ejercicios correctamente resueltos y el tiempo que ha tardado el estudiante en realizar cada tarea. Esta información está disponible, al menos hasta que el curso se dé por concluido. Al final de cada sesión, la información relativa a las acciones del estudiante se almacena en una base de datos a la que el profesor puede acceder. En posteriores sesiones, el sistema extraerá la información de esa base de datos, para reconstruir la situación y tener disponible de nuevo información sobre las acciones previas del estudiante.

\subsection{EL PERFIL DEL ESTUDIANTE}

El perfil del estudiante afecta al proceso de guía en dos sentidos. En primer lugar, influye en la generación dinámica de los itinerarios ofrecidos a cada estudiante. El sistema, en el momento de evaluar qué reglas se activan en cada momento, debe comprobar las condiciones de activación de dichas reglas. Estas condiciones pueden incluir requisitos relacionados con alguna característica del estudiante, por lo que el sistema debe consultar los datos almacenados sobre el perfil del estudiante, y compararlos con los que aparecen en la condición de activación.

Por otra parte, durante la generación de las páginas HTML asociadas a una tarea, el sistema consulta los datos del perfil del estudiante para compararlos con las características de los elementos multimedia disponibles relacionados con esa tarea. A continuación, selecciona los más apropiados para ese estudiante concreto, construyendo con ellos las páginas que se mostrarán a continuación. Pueden encontrarse más detalles sobre el proceso de adaptación en TANGOW en (Carro, 1999b).

\section{ANALISIS DE OTROS SISTEMAS EXISTENTES}

Las investigaciones y resultados en torno a los sistemas hipermedia adaptativos han adquirido especial relevancia en los últimos años (AH\&H, 1998; AS\&UM, 1999; AH, 2000).

Dentro del ámbito nacional merece la pena mencionar el proyecto SEBASTIÁN (Fernández, 1998), que se propone desarrollar una herramienta de ayuda a la creación de cursos basados en Internet. También son relevantes los estudios sobre la integración de tutores inteligentes y sistemas hipermedia para crear sistemas hipermedia adaptativos (Pérez, 1995).

En el marco europeo, uno de los trabajos más interesantes es el relacionado con la generación de presentaciones hipermedia a partir de consultas a bases de datos y el uso de hipertexto adaptativo para la enseñanza a través de Internet (de Bra, 1999). Esta investigación se materializa en AHA (de Bra, 1998), sistema que permite la generación de cursos adaptativos para la Web. En AHA, la adaptación se realiza mediante la inclusión de condiciones como comentarios en las páginas HTML. Estas condiciones actúan como filtros para decidir qué porciones de la página se muestran al estudiante. La principal diferencia con TANGOW es que, en este último, las páginas HTML son creadas desde cero, enlazando los elementos multimedia más apropiados para cada estudiante, mientras que en AHA las páginas ya están previamente creadas y se decide qué porciones de ellas se muestran y cuáles no.

Hasta la fecha, la mayoría de los sistemas de enseñanza adaptativos fundamentan su adaptatividad en características estructurales, que varían según el perfil y, en algunos casos, según las acciones de los estudiantes. En esta línea se encuentra el trabajo de da Graça (1998), donde se utiliza SGML para definir la estructura general de los documentos, especificando después cuáles serán los elementos concretos que aparecerán en esa estructura. En TANGOW no es necesario definir distintas estructuras para las páginas 
HTML, pues cada página se crea justo antes de presentarla al estudiante, eligiendo, de entre los elementos multimedia asociados a la tarea activa, aquéllos más apropiados para ese estudiante concreto.

Otra propuesta consiste en la representación de la estructura mediante grafos de requisitos, donde cada nodo es un concepto de un curso y cada enlace une un concepto con los conceptos que deben haber sido asimilados previamente. Este tipo de aproximación es utilizada por Lai (1995), Nykinen (1997), da Silva (1998), Garlatti (1999) y Henze (1999). En todos estos casos, existe una relación directa entre los contenidos y la estructura de la información. Es posible que distintos estudiantes visiten nodos diferentes del grafo, pero la información asociada a cada nodo sea siempre la misma. Esto difiere de TANGOW, donde los documentos asociados a cada tarea son generados dinámicamente, y los contenidos presentados a dos estudiantes que están realizando la misma tarea pueden variar dependiendo del perfil de los mismos.

Otro sistema de enseñanza a través de Internet que merece la pena mencionar es SKILL (Neumann, 1998). En SKILL el curso se estructura de forma similar a los modelos de grafo de requisitos anteriores, pero la adaptatividad del sistema se implementa teniendo en cuenta los conocimientos previos de cada estudiante. En TANGOW no sólo se puede tener considerar este factor, sino también otras características propias de cada perfil, como el lenguaje elegido para el curso o la edad del estudiante, que puede condicionar el tipo de documentación que se le ofrezca. Por otra parte, SKILL, al igual que TANGOW, almacena los resultados de cada sesión, que también se utilizarán en sesiones posteriores. Una de las principales diferencias entre SKILL y otros sistemas es que éste incorpora la posibilidad de realizar anotaciones, que permiten la realización de algunas actividades de colaboración entre usuarios del sistema.

Una aproximación distinta es la propuesta en el sistema DCG (Vassileva, 1997, 1998), donde los contenidos se estructuran como un mapa de carreteras utilizado para generar el plan del curso, plan que es revisado cada vez que es necesario adaptar el curso al estudiante concreto. La principal diferencia entre DCG y TANGOW es que en el primero, cada vez que es necesario adaptar el curso a las características del estudiante, debe revisarse todo el plan, mientras que en TANGOW dicha adaptación se realiza en cada paso del proceso, y sólo implica la selección de un conjunto de tareas activables en cada momento.

Directamente relacionado con la estructuración de los cursos, está el mantenimiento de los mismos. La separación de la estructura del curso con respecto a los contenidos es una buena medida a tomar para facilitar esta tarea, como proponen de Bra (1999) y Jun (1999). En este sentido, hay sistemas que mantienen la consistencia de los enlaces dentro de las páginas y que automatizan la estructuración de las mismas, como Hyper-G (Andrews, 1995). En cualquier caso, una forma efectiva de mantener los cursos es produciendo módulos que puedan ser reutilizados en distintas partes del mismo curso, como sugiere Dietinger (1999).

En la actualidad existen algunas herramientas desarrolladas para la edición gráfica de contenidos. Algunas pueden catalogarse como herramientas de autor para productos multimedia, tales como Macromedia Director ${ }^{\circledR}$, o como editores de páginas web (FrontPage ${ }^{\circledR}$, HotMetal ${ }^{\circledR}$, o Netscape Composer $\left.{ }^{\circledR}\right)$, pero ninguna de ellas incluye un mecanismo para incluir adaptación en los cursos desarrollados.

Desde el punto de vista de la colaboración en los sistemas educativos, una de las tendencias es facilitar que los estudiantes compartan los conocimientos para compaginar el conocimiento colectivo con las aportaciones individuales (Verde- jo, 1998). Se han realizado algunos trabajos relacionados con la creación de en- tornos de enseñanza colaborativos accesibles a través de Internet (Hmelo, 1998; Maly, 1997). También se ha analizado la viabilidad de utilizar la Web como el marco de construcción de estas aplicaciones, observándose que el tiempo de desarrollo y evaluación de estos sistemas es muy largo (Nicol, 1999).

\section{CONCLUSIONES Y TRABAJO FUTURO}

El sistema TANGOW permite la creación de cursos adaptativos accesibles a través de Internet, mediante la descripción de tareas y reglas docentes, que permiten guiar a los estudiantes durante su proceso de aprendizaje. Este proceso de guía consiste en adaptar, tanto los conceptos presentados en cada momento, como los contenidos concretos de las páginas HTML, teniendo en cuenta el perfil del estudiante, las acciones que ha realizado durante la ejecución del curso y la estrategia de aprendizaje seleccionada. 
Con TANGOW es posible satisfacer las necesidades de distintos tipos de estudiantes sin tener que crear un curso por cada preferencia, estilo de aprendizaje o evolución de los estudiantes. Además, es posible presentar un determinado concepto con distintos niveles de detalle, de modo que los estudiantes puedan estudiar los conceptos de forma general o profundizar en ellos.

El diseño modular de los cursos, basado en la definición de tareas y reglas docentes, y en la clasificación de elementos multimedia, junto con el almacenamiento de estos componentes en bases de datos, permite reducir el coste de mantenimiento, ya que los profesores pueden añadir, eliminar o modificar componentes fácilmente. Además, esto hace posible la reutilización de tareas, reglas y elementos multimedia en distintas partes del curso o en distintos cursos.

Una ventaja adicional del sistema TANGOW es que el servidor de cursos es accesible a través de Internet mediante cualquier navegador estándar, por lo que los estudiantes no necesitan ningún software adicional.

En cuanto a trabajo futuro, uno de nuestros primeros objetivos consiste en incorporar ejercicios de respuesta libre que aumenten la versatilidad del sistema y permitan considerar criterios pedagógicos específicos.

También se pretende mejorar los mecanismos de adaptación, incluyendo la posibilidad de cambiar la estrategia de aprendizaje activa de forma dinámica, en función de los resultados obtenidos por el estudiante.

Por otra parte, la arquitectura de TANGOW es apropiada para incluir facilidades de colaboración entre los usuarios del sistema. Sería factible que ios tutores consultaran la información sobre las acciones de los estudiantes, para poder ofrecerles sugerencias en tiempo real. También seria deseable la comunicación entre estudiantes para compartir conocimientos, y la inclusión de actividades colaborativas supervisadas por el profesor.

Desde el punto de vista del profesor, el carácter dinámico y adaptativo de los cursos hace que su estructura sea difícil de abarcar mentalmente en tiempo de diseño. El curso como tal no existe, ya que depende del perfil del alumno y del contexto de ejecución. Convendría, por ello, extender la herramienta de diseño que se está utilizando en la actualidad, para proporcionar una visión global sobre las posibles estructuras del curso para los distintos perfiles de estudiantes, e incluir mapas donde se visualicen las relaciones entre los distintos componentes.

Otro objetivo, planteado más a largo plazo, consiste en proponer un método que permita diseñar cursos adaptativos a partir del material utilizado para la impartición de cursos tradicionales.

Por último, actualmente se están diseñando nuevos cursos que serán puestos a disposición de estudiantes de Ingeniería Informática de la Universidad Autónoma de Madrid, y cuya experimentación permitirá realizar una evaluación de la efectividad de TANGOW.

\section{REFERENCIAS BIBLIOGRÁFICAS}

AH\&H (1998). 2nd Workshop Qn Adaptive Hypertext and Hypermedia, celebrado junto con HYPERTEXT'98. Pittsburg, USA. Proceedings disponibles en: http://wwwis.win.tue.nl/asum99/

AH (2000). International Con ference Qn Adaptive Hypermedia and Adaptive Web-based Systems. Trento, Italia, Agosto 2000. http://AH2000.itc.it/

ANDREWS, K., KAPPE, F., MAURER, H. (1995). The Hyper-G Network Information System. Journal of Universal Computer Science, 4 (1), 206-220.

AS\&UM (1999). 2nd Workshop Qn Adaptive Systems and User Modeling on the World Wide Web, celebrada junto con la Eight International World Wide Web Conference y la 7-th International Conference on User Modeling. Banif, Canadá, Junio 1999. Proceedings disponibles en: http://wwwis.win.tue.nl/asum99/ 
BRUSILOVSKY, P. (1997). Distributed intelligent tutoring on the Web. Proceedings de 8th World Con ference of the AIED Society. Kobe, Japón. Disponible en http://www.contrib.andrew.cmu,edu/plb/AIED97_workshop/Brusilovsky/Brusilovsky.html

BRUSILOVSKY, P., ANDERSON, J. (1998). ACT-R electronic bookshelf: An adaptive system for learning cognitive psychology on the Web. Proceedings de la conferencia WebNet-98, AACE. Orlando, Florida. 92-97.

CARRO, R.M., PULIDO, E., RODRÍGUEZ, P. (1999a). TANGOW: Un Sistema de Enseñanza Adaptativa a través de Internet. Actas del Congreso Nacional de Informática Educativa CONIED’99. Puertollano, Ciudad Real.

CARRO, R.M., PuLIDO, E., RODRÍGUEZ, P. (1999b). Dynamic generation of adaptive Internet-ha- sed courses. Journal of Network and Computer Applications, 22, 249-257.

CARRO, R.M., PULIDO, E., RODRÍGUEZ, P. (1999c). Designing Adaptive Web-based Courses with TANGOW, en Cumming, G., Okamoto, T., Gomez, E. Advanced Research in Computers and comunications in Education, (2). Amsterdam: TOS Press, 697-704.

CARRO, R.M., PULIDO, E.:RODRIGUEZ, P. (2000). TANGOW: a Model for Internet Based Learning. International Journal of Continuing Engineering Education and Life-Long Learning, special Issue on «Internet based learning and the future of education». Próxima publicación

DA GRAÇA, M., BENEDITO, J., PONTIN, R. (1998). Tools for Authoring and Presenting Structured Teaching Material in the WWW. Proceedings de la conferencia WebNet-98, MCE. Orlando, Florida. 194199.

DA SILVA, D.P, VAN DURM, R., DUvAL, E., Ouvi, H. (1998). Concepts and documents for adaptive educational hypermedia: a model and a prototype. Proceedings del Second Workshop Qn Adaptive Hypertext and Hypermedia, en la Ninth ACM Conference on Hypertext and Hypermedia. Pittsburgh, USA. 35-43.

DE Bi, P., CALVI, L. (1998). AHA: A Generic Adaptive Hypermedia System. Proceedings del Second Workshop on Adaptive Hypertext and Hypermedia, en la Ninth ACM Conference on Hypertext and Hypermedia. Pittsburgh, USA. 5-11.

DE Bu, P. (1999). Design Issues in Adaptive Web-Site Development. Proceedings del Second Workshop on Adaptive Systems and User Modeling on the WWI'V en la 7th International Conference on User Modeling. Banif, Canadá. 29-39.

DIETINGER, T. ET AL. (1999). An Associative Repository for the Administration of Course Modules. Proceedings de la conferencia WebNet-99, MCE. Honolulu, Hawaii. 295-300.

FERNÁNDEZ, M.C., SANTACRUZ, L., TORRES, L., DELGADO, C. (1998). SEBASTIÁN: Una propuesta Tecno-Pedagógica para la Educación Virtual en Internet. Actas del II Congreso Internacional sobre Comunicación, Tecnología y Educación. Oviedo, España.

GARLATTI, S., IKSAL, S., KERVELLA, P. (1999). Adaptive on-line information system by means of a task model and spatial views. Proceedings del Second Workshop on Adaptive Systems and User Modeling on the Web en la 8th International WWW Conference. Toronto, Canada. Disponible en http://www.contrib.andrew.cmu.edu! .-p1bíWWWUM99_workshop/garlat- ti/garlatti.html

HENZE, N., NEJDL, W. (1999). Adaptivity in the KBS Hyperbook system. Proceedings del Second Workshop on Adaptive Systems and User Modeling on the Web en la 8th International WWW Conference. Toronto, Canada. Disponible en http://www.contrib.andrew.cmu.edul.plb/WWWUM99_workshop/henze/ henze.html 
HMELO, C., GUZDIAL, M., TURNS, J. (1998). Computer-Support for Collaborative Learning: Learning to Support Student Engagement. AACE Journal of Interactive Learning Research, 9 (2).

JUN, Y., KIM, D. (1999). An organizational tool for construcing and presenting multimedia teaching materials, en Cumming, G., Okamoto, T., Gomez, L. Advanced Research in Computers and comunications in Education, (1). Amsterdam: lOS Press, 171-174.

LAI, M.C., CHEN, B.H., YuAN, S.M. (1995). Toward a New Educational Environment. Proceedings de la 4th International World Wide Web Conference. Boston, USA. Disponible en http://www.w3.orglpub/ConferencesfWWW4/Papers/238/

MALY, K., ABDEL-WAHAD, H., OVERSTREET, C., WILD, J., GUPTA, A., YOUSSEF, A., ST0IcA, E., ALSHAER. (1997). Interactive distance learning over intranets. IEEE Internet Comput. , 1(1), 67-71.

NEUMANN, G., ZIRVAS, J. (1998). SKILL: A Scalable Internet-Based Teaching and Learning System, Proceedings de la conferencia WebNet-98, MCE. Orlando, Florida. 688-693.

NICOL, J., GUTFREUND, Y., PASCHErrO, J., RUSH, K., MARTIN, C. (1999). How the Internet Helps Build Collaborative Multimedia Applications. Communications of the ACM, 42 (1), 79-85.

NYKÄNEN, 0. (1997). User Modeling in WWW with Prerequisite Graph Model. Proceedings del Workshop on Adaptive Systems and User Modeling on the World Wide Web en la Sixth International Conference on User Modeling. Chia Laguna, Sardinia.

PÉREZ, T.A., GUTIÉRREZ, 3., LOPISTÉGUY, P. (1995). An Adaptive Hypermedia System. Proceedings de la conferencia Artificial Intelligence in Education. Charlottesville, EE.UU.

SUÁREZ, A., PULIDO, E., CMuo R. (2000). An Adaptive Web-based Course in Financial Engineering. Proceedings de EDMEDIA 2000. Montreal, Canada. Próxima publicación.

VASSILEVA, J. (1997). Dynamic Courseware Generation on the W'vVW. Proceedings de la 8th World Conference of the AIED Society. Kobe, Japón. 498-505.

VASSILEVA, J. (1998). A Task-Centred Approach for User Modeling in a Hypermedia Office Documentation System, en Brusilovsky, P., Kobsa, A. and Vassileva J. Adaptive Hypertext and Hypemiedia. Dordrecht: Kluwer Academic Publisher, 209-247.

VERDEJO, M.F., BAKROs, B, ABAD, M.T. (1998). Supporting Distance Learners For Collaborative Problem Solving. Proceedings del ED_MEDIA \& ED-TELECOM 98 de la AACE. Freiburg, Alemania. 1407-1412.

WEBER, G., SPECHT, M. (1997). User modeling and adaptive navigation support in WWW-based tutoring systems. Proceedings de la conferencia User Modeling’97. Italia. 289-300.

\section{PERFIL ACADÉMICO Y PROFESIONAL DE LOS AUTORES}

Rosa María Carro Salas es profesora ayudante en la Escuela Técnica Superior de Informática de la Universidad Autónoma de Madrid. Obtuvo su título de Ingeniera en Informática por la Universidad Autónoma de Madrid en 1997. Actualmente está realizando su tesis doctoral en el campo de la generación dinámica de cursos de enseñanza adaptativos para Internet. Su línea de investigación se centra en los entornos de enseñanza adaptativos, sistemas de aprendizaje en la Web y multimedia en la educación.

Estrella Pulido Cañabate es profesora titular en la Escuela Técnica Superior de Informática de la Universidad Autónoma de Madrid. Obtuvo su título de Doctora en Informática por la Universidad de Bristol en 1996. Su línea de investigación actual se centra en los sistemas de ayuda a la enseñanza 
basados en la Web, los sistemas de hipertexto adaptativo, los sistemas colaborativos y la multimedia en la educación.

Pilar Rodríguez Marín es profesora titular en la Escuela Técnica Superior de Informática de la Universidad Autónoma de Madrid. Obtuvo su título de Doctora en Informática por la Universidad Complutense de Madrid en 1990. Trabajó para IBM desde 1985 hasta 1996. Su línea de investigación se centra en el área de los sistemas adaptativos, la enseñanza a través de la Web y la multimedia educativa.

Las tres autoras de este artículo participan actualmente en los proyectos "InterEdu: Internet en la Educación (TEL97-0306)” y Desarrollo de un entorno avanzado para la docencia en red”, financiados por la CICyT y la UAM respectivamente.

Universidad Autónoma de Madrid

Escuela Técnica Superior de Informática

Ctra. Colmenar Viejo, Km. 15. Campus de Cantoblanco.

28049 Madrid. España

Tlfno.: 9134822 76, 9134822 89, 913482283

Fax: 913482235

Correo eléctronico:

Rosa.Carro@ii.uam.es

Estrella.Pulido@ii.uam.es

Pilar.Rodríguez@ii.uam.es 